\section{Melanom: PD1-Checkpointinhibitor verlängert das progressionsfreie Überleben}

\begin{abstract}
In der Phase-III-Studie CheckMate 067 wurden der PD1-Checkpointinhibitor Nivolumab allein, der CTLA-4-Inhibitor Ipilimumab allein sowie die Kombination beider Substanzen beim metastasierten Melanom verglichen.
\end{abstract}

$\mathrm{D}$ ie 945 bisher unbehandelten Patienten mit nicht resezierbarem Melanom im Stadium III oder IV erhielten Nivolumab allein, in Kombination mit Ipilimumab oder Ipilimumab allein. Primäre Endpunkte waren progressionsfreies Überleben und Gesamtüberleben.

Das mediane progressionsfreie Überleben war unter der Therapie mit Nivolumab allein oder in Kombination mit Ipilimumab signifikant länger als unter Ipilimumab allein. Es lag bei 11,5 Monaten unter der Kombinationstherapie (Hazard Ratio [HR] 0,42; $<<0,001$ ) bzw. 6,9 Monaten unter Nivolumab allein (HR vs. Ipilimumab 0,57; p < 0,001) im Vergleich $\mathrm{zu}$ 2,9 Monaten unter Ipilimumab allein. Die HR für die Kombina- tionstherapie versus Nivolumab allein betrug 0,74. Auch die Ansprechraten waren in den Nivolumab-Armen höher als unter Ipilimumab allein.

Bei Patienten mit positivem PD-L1 („programmed death-ligand 1“)-Status betrug das mediane progressionsfreie Überleben 14,0 Monate sowohl unter der Kombinationstherapie als auch unter Nivolumab allein und 3,9 Monate unter Ipilimumab allein. Bei Patienten mit PD-L1negativen Tumoren war die Kombination aus PD1- und CTLA-4-Blockade jedoch effektiver als die alleinige Therapie mit nur einer dieser Substanzen. Das progressionsfreie Überleben betrug hier 11,2 Monate unter der Kombinationstherapie und 5,3 Monate unter Nivolumab allein.
Die häufigsten unerwünschten Ereignisse unter der Kombinationstherapie waren Diarrhö (44,1\%), Fatigue (35,1\%) und Juckreiz (33,2\%). Therapiebedingte unerwünschte Ereignisse vom Grad 3 oder 4 traten bei 16,3\% der Patienten unter Nivolumab allein, bei $55 \%$ der Patienten unter der Kombinationstherapie und bei 27,3\% der Patienten unter Ipilimumab allein auf.

Fazit: Bei Patienten mit bisher unbehandeltem metastasiertem Melanom führte die Therapie mit Nivolumab allein oder in Kombination mit Ipilimumab zu einer signifikanten Verlängerung des progressionsfreien Überlebens im Vergleich zur Therapie mit Ipilimumab allein. Da die unerwünschten Ereignisse unter der Kombinationstherapie handhabbar sind, kann diese Therapie bei einem breiten Spektrum klinischer Situationen eingesetzt werden.

Judith Neumaier

Larkin J et al. Combined Nivolumab and Ipilimumab or Monotherapy in Untreated Melanoma. N Engl J Med. 2015;373(1):23-34.

\title{
Ipilimumab bei Melanom: Patienten auf Nebenwirkungen vorbereiten
}

\section{Troy Horvat und Kollegen vom Memorial Sloan Kettering Cancer Center in New York fassten ihre Erfahrungen mit Ipilimumab, die sie außerhalb von klinischen Studien gemacht haben, zusammen.}

\footnotetext{
er Anti-CTLA-4-Antikörper Ipilimumab ist der erste Checkpointinhibitor, für den bei metastasiertem $\mathrm{Me}$ lanom eine Verlängerung des Überlebens nachgewiesen wurde. Allerdings treten häufig immunvermittelte, unerwünschte, zum Teil schwerwiegende, Ereignisse auf. Das bestätigte sich auch in einer retrospektiven Analyse der Daten von 298 Patienten (medianes Alter 65 Jahre) mit Melanom, die zwischen April 2011 und Juli 2013 mit Ipilimumab in der Standarddosierung von $3 \mathrm{mg} / \mathrm{kg}$ Körpergewicht behandelt worden waren.
}

$85 \%$ der Patienten hatten immunvermittelte unerwünschte Ereignisse. Diese waren bei $31 \%$ vom Grad 3, bei $7 \%$ vom Grad 4 und bei 1 Patienten tödlich. Am häufigsten war eine Diarrhö (14\%). 35 \% der Patienten benötigten systemische Kortikosteroide, $10 \%$ darüber hinaus eine Anti-TNF- $\alpha$-Therapie. 19\% der Patienten brachen die Therapie wegen solcher Ereignisse ab.

Das mediane Gesamtüberleben betrug 16,5 Monate, die 2-Jahres-Überlebensrate $39 \%$. Die Zeit bis zum Therapieversagen, definiert als Beginn einer neuen Therapie oder Tod, betrug median 5,7 Monate. $12 \%$ der Patienten erreichten eine langfristige Krankheitskontrolle ohne weitere Antitumortherapie, d.h. bei $88 \%$ kam es zu Progression, Therapieumstellung oder Tod.

Gesamtüberleben und Zeit bis zum Therapieversagen wurden durch das
Auftreten immunvermittelter unerwünschter Ereignisse oder die Notwendigkeit systemischer Kortikosteroide nicht beeinflusst.

Fazit: Immunvermittelte unerwünschte Ereignisse treten unter Ipilimumab häufig auf, allerdings werden diese vermutlich in verschiedenen Ländern unterschiedlich beurteilt. Nach Erfahrung des Teams um Horvat benötigt etwa ein Drittel der Patienten systemische Kortikosteroide, und fast ein Drittel dieser Patienten benötigt eine weitere Immunsuppression. Ärzte und Patienten sollten auf solche Ereignisse vorbereitet sein und deren Behandlung frühzeitig beginnen. Außerdem sollten sie wissen, dass dadurch Gesamtüberleben und Zeit bis zum Therapieversagen nicht beeinträchtigt werden.

Judith Neumaier

Horvat TZ et al. Immune-Related Adverse Events, Need for Systemic Immunosuppression, and Effects on Survival and Time to Treatment Failure in Patients With Melanoma Treated With Ipilimumab at Memorial Sloan Kettering Cancer Center. J Clin Oncol. 2015;33(28):3193-8. 